\title{
Throughput in cooperative wireless networks
}

\author{
Diego Armando Giral Ramirez, Cesar Hernandez, Fredy Martinez
}

Technological Faculty, Universidad Distrital Francisco Jose de Caldas, Colombia

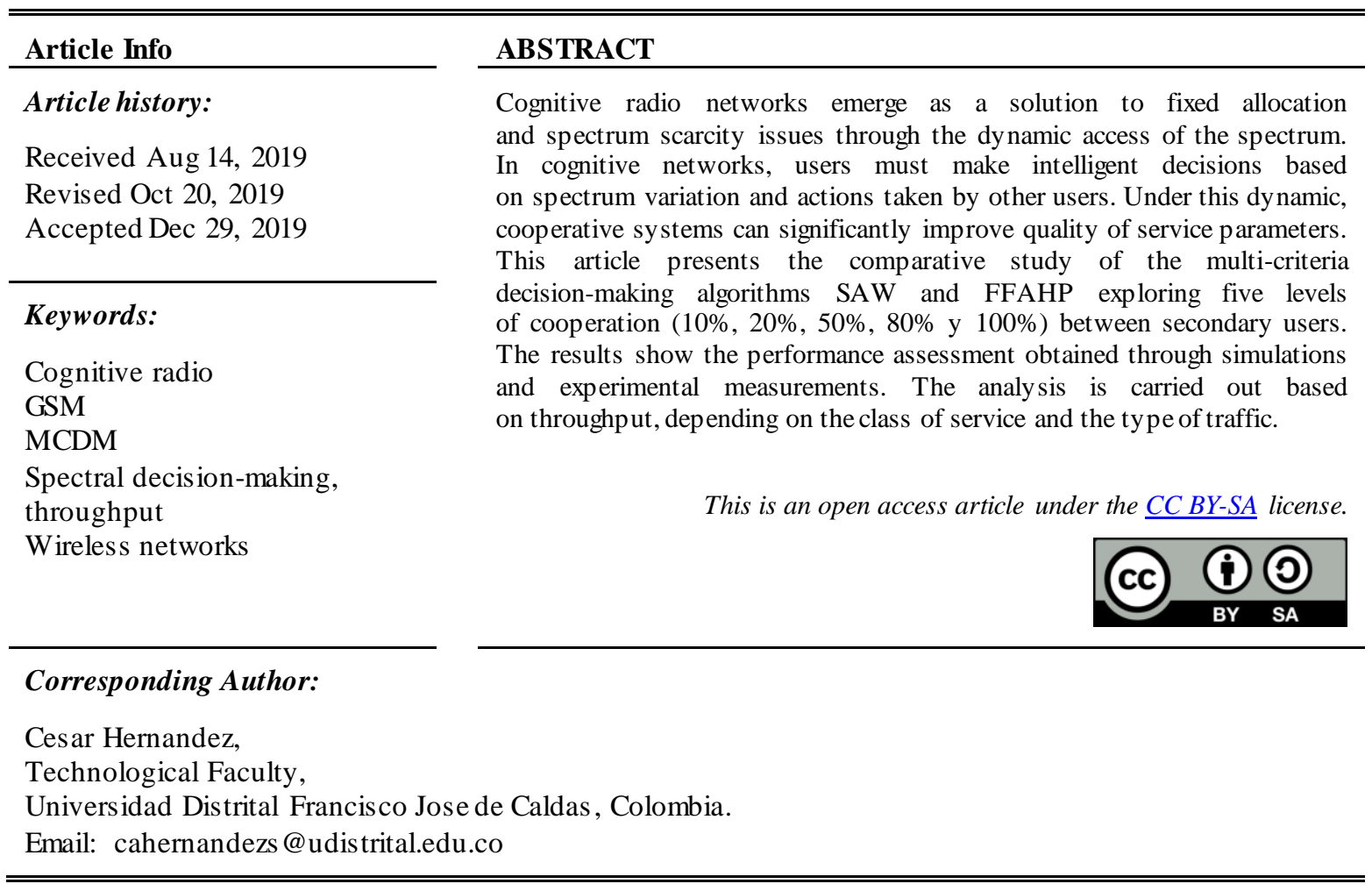

\section{INTRODUCTION}

The growth of wireless applications proposes new challenges in the future of communication systems [1-3]. Fixed allocation policies, the exponential growth of the demand, the scarcity of the radioelectric spectrum and its underuse are important issues in wireless networks and have prompted strategies for the dynamic and optimal access of the spectrum as as a solution [4]. Cognitive Radio (CR) is a relatively new field that proposes efficient and adaptive methodologies for the dynamic allocation of the existing radio spectrum [4]. Cognitive radio detects its environment and adjusts its operation parameters in a dynamic and autonomous manner seeking to modify the system. Techniques based in CR allow to maximize performance, reduce interference and facilitate interoperability [5-7].

In contrast with traditional networks, there are two types of users: the user that pays for a licensed frequency band known as the primary user (PU) and the non-licensed user known as the secondary user (SU), that makes an opportunistic use of the available licensed spectrum [8-9]. The purpose of a cognitive radio network (CRN) consists on granting access to the SU to the available frequency band, without generating interference to the PU [8-10]. This is achieved through a management model called cognitive cycle that is characterized by four main functions: spectrum detection, spectrum decision, spectral mobility and spectrum sharing.

The focus of this research consists on carrying out the decision-making process of a descentralized cognitive radio system by granting the nodes the capacity to learn from the environment and proposing strategies that allow SU to exchange information cooperatively. While multiple techniques are available for the analysis of CR, some cooperative algorithms that are striving in cognitive structure-based applications. CR and cooperative strategies have delivered new models for the efficient use of radio networks. For instance, the cooperative decision-making process allows users to communicate between them 
to exchange interference measurements captured locally. The goal is to harness spatial diversity relying on the unlicensed user that shares detection information with neighbouring users [11-12].

The proposed solution is based in cooperative CRN through the exchange of information between $\mathrm{SU}$, that can increase transmission speeds and significantly improve the quality parameters of service, latency, throughput, reliability, signalization, PU interference, energetic efficiency, bandwidth (BW), signal-to-noise-plus-interference-ratio (SINR) and bit error rate (BER) [13-19]. The results of this article show the performance assessment obtained through simulations with experimental measurements of the comparative study of two multi-criteria decision-making algorithms: SAW and FFAHP. The analysis uses a power matrix as database, which is segmented into five levels of cooperation $(10 \%, 20 \%, 50 \%, 80 \%$ and $100 \%$ ) between secondary users. The frequency band corresponds to the GSM technology. The analysis is determined based on throughput according to the service class (real time-RT and better effort-BE) and the type of traffic (high traffic-HT and low traffic-LT).

\section{RESEARCH METHOD}

For the comparative analysis of the decision-making multicriteria strategies, a simulator was developed that uses an information database comprised of 551 channels. For training and validation, the Test-Validation technique is used with an $83 \%-17 \%$ ratio that corresponds to 10800 training data and 1800 validation data. This is equivalent to one hour of training and 10 minutes of assessment. The information corresponds to real data captured in a previous metering campaign within the GSM frequency band.

Figure 1 presents the general structure of the implemented model. The simulator includes four processing blocks: the first one is called "Collaborative" which segments the power matrix and distributes it among SU, the second one is the "MCDM" block that includes the mathematical models for decision-making, the third one is the "Search Algorithm" which is a search structure in charge of simulating and quantifying throughput characteristics. Finally, the "Figure" block builds the respective charts.

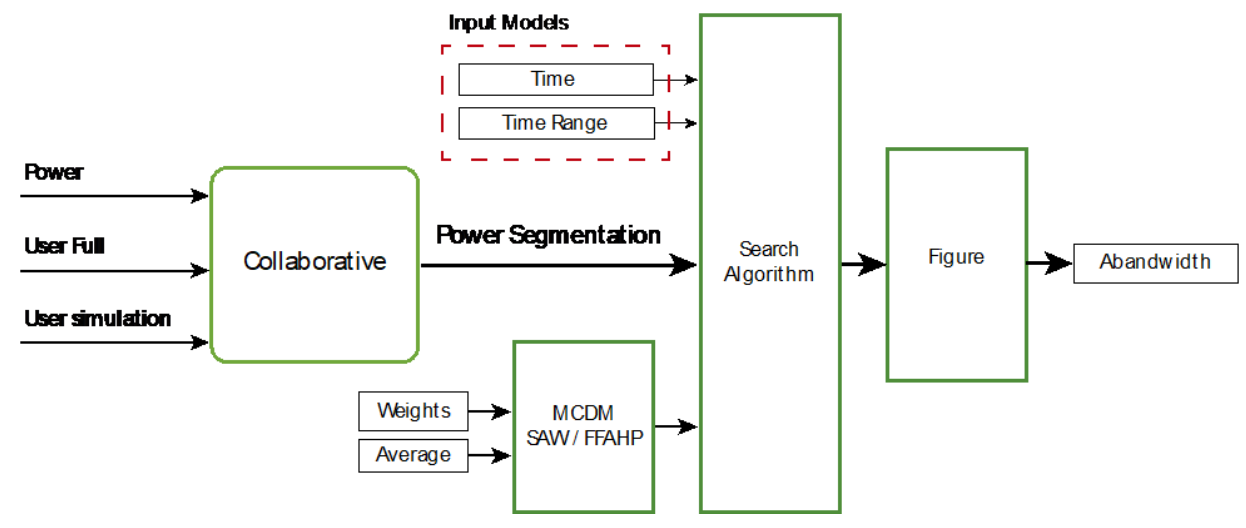

Figure 1. General structure of the model

\subsection{Algorithms for spectrum allocation}

Both multi-criteria decision-making algorithms (MCDM) chosen for Feedback Fuzzy Analytical Hierarchical Process (FFAHP) and Simple Additive Weighting (SAW).

\subsubsection{FFAHP}

Fuzzy logic is a particularly adequate tool to make decisions in scenarios where the inputs are generally uncertain or imprecise. In essence, the Fuzzy Analytical Hierarchical Process (FAHP) uses the same methodology of the AHP algorithm. However, fuzzy logic is helpful in dealing with the subjectivity and uncertainty generated during the assessment process. In the FAHP algorithm, the weight vector after normalization is given by (1).

The Feedback FAHP (FFAHP) proposes the feedback of information taken from previous assessments based on the FAHP method. In methods based in AHP, judgment matrices are needed to carry out comparative assessments that determine the level of relative importance of each combined pair of criteria, sub-criteria and alternatives independently. For instance, real time (RT) and better effort (BE) applications have different approaches. For RT, the sub-criteria with the highest priorities are those that reduce delay, 
such as the probability of availability (PA) and the average availability time (AAT). For BE, the sub-criteria with the highest priorities are those that increase data rates such as bandwidth (BW) and SINR.

$$
\mathrm{W}=\left(\mathrm{d}_{1}, \mathrm{~d}_{2}, \ldots, \mathrm{d}_{\mathrm{n}}\right)^{\mathrm{T}}=\left(\frac{\mathrm{d}_{1}}{\sum_{\mathrm{i}=1}^{\mathrm{n}} \mathrm{d}_{\mathrm{i}}}, \frac{\mathrm{d}_{2}}{\sum_{\mathrm{i}=1}^{\mathrm{n}} \mathrm{d}_{\mathrm{i}}}, \ldots, \frac{\mathrm{d}_{\mathrm{n}}}{\sum_{\mathrm{i}=1}^{\mathrm{n}} \mathrm{d}_{\mathrm{i}}}\right)
$$

If the algorithm is using a RT application, the score for each channel is computed using (2), while (3) is used in BE applications. Table 1 shows a description for each acronym used in (3) and (4).

$$
\begin{aligned}
& \text { Score }_{R T}=0,3593(P D)+0,2966(T E D)+0,1970(P S I N R)+0,1471(P W A) \\
& \text { Score }_{B E}=0,1607(P D)+0,1523(T E D)+0,3949(\operatorname{PSINR})+0,2921(P W A)
\end{aligned}
$$

Table 1. Vectors for multi-criteria analysis

\begin{tabular}{lll}
\hline Acronym & \multicolumn{1}{c}{ Average } & \multicolumn{1}{c}{ Description } \\
\hline PD & Availability probability & Average value for each column of the availability matrix \\
TED & Average availability time & Average of consecutive values of the availability matrix \\
PSINR & Average SINR & Average value from each column of the SINR without including zeros \\
PWA & Average bandwidth & Average of each column in the bandwidth matrix \\
\hline
\end{tabular}

\subsubsection{SAW}

This algorithm develops a decision matrix that includes both criteria and alternatives. For each intersection of the matrix, the algorithm assigns a weight according to the criteria of the designer. This allows to establish a score for each SO assessed as well as a ranking of alternatives. The SO with the highest score is selected through (4) [20-22] where $r_{i, j}$ belongs to the matrix and the sum of weights is equal to 1 .

$$
u_{i}=\sum_{j=1}^{M} \omega_{i} r_{i, j} \quad \forall i \in 1, \ldots, N
$$

The steps required to develop this algorithm are: (1) identifty the objectives and alternatives; (2) assess the alternatives; (3) determine the steps of each combination; (4) add new values according to preferences; and (5) analyze sensitivity [23-25].

\subsection{Simulation structure}

In this section, a detailed description is given on each module described in Figure 1 based on input-output variables and their corres ponding pseudo-code.

\subsubsection{Cooperative model}

The "Collaborative" module is in charge of carrying out the segmentation of the power matrix among SU, the parametrization is carried out through three input variables: the power matrix and the number of available users. The latter is computed depending on the size of the power matrix and the number of users included in the analysis. Figure 2 presents the structure of the input variables defined for this module. It only has a single input variable, which corresponds to the segmented power matrix.

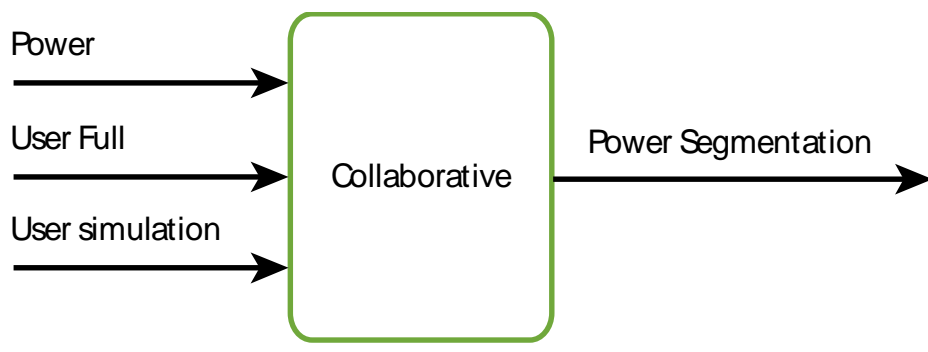

Figure 2. Inputs and outputs of the collaborative model 
For the implementation of the module, three functions from Matlab are used. The following pseudo-code presents a summary of the matrix segmentation structure. The general structure consists on randomly determining the location of users participating in the simulation. The size of the information is equal for all users.

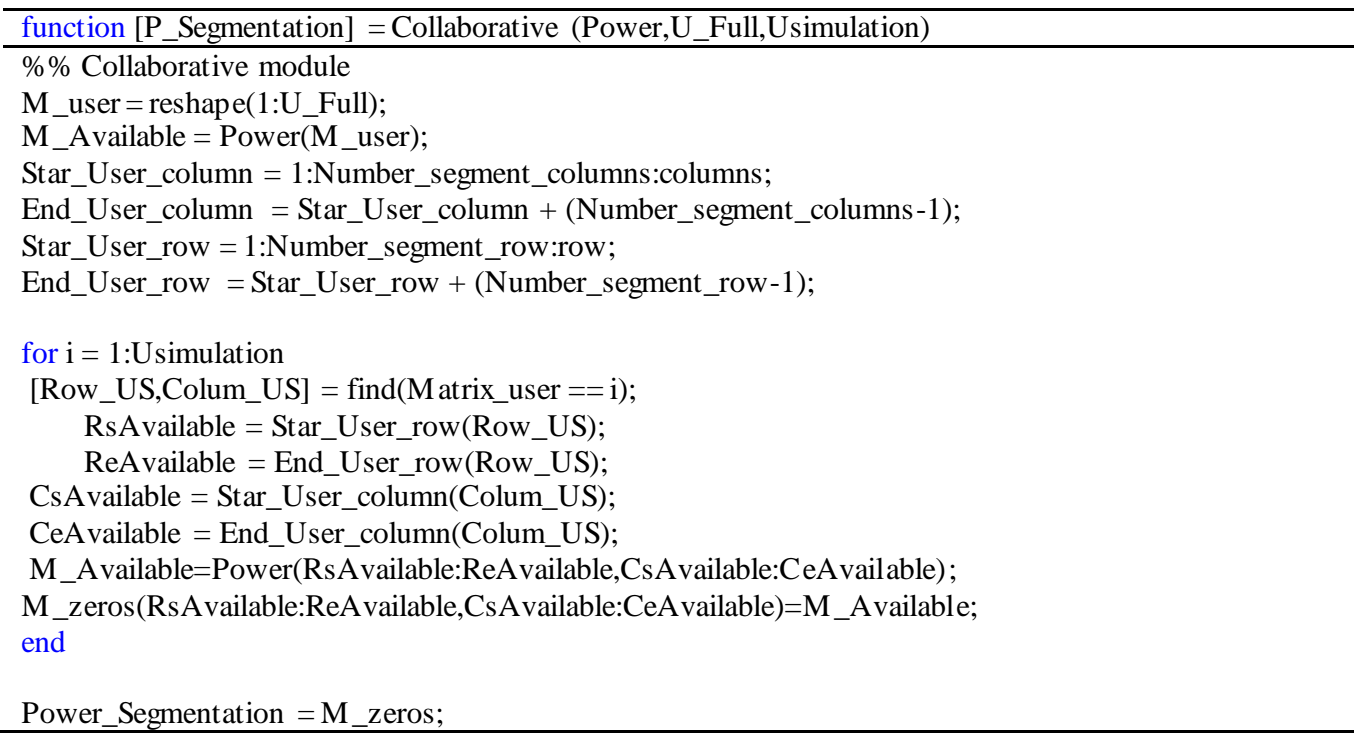

\subsubsection{Multi-criteria functions}

The SAW and FFAHP methods require the same input and output variables. The variation of each strategy lies in the mathematical structure shown in the block diagram in Figure 3. The input parameters correspond to a vector containing the average values associated to the availability of channels. The elements of said vector are defined as PD, TED, PSINR and PWA. The description of each acronym is presented in Table 1. The size of each vector is $l \times n$ where $n$ corresponds to the number of columns of the segmented power matrix. The second input of the multi-criteria module is a vector defined as "Weight". Each element of this vector includes the weight and/or score of each variable defined in the "Average" vector.

Based on the assigned weights and average values of PD, TED, PSINR and PWA, the multi-criteria module determines score and ranking of each channel. These characteristics correspond to the output variables of the multi-criteria module Figure 3. The purpose of this method is to create a vector that classifies channels according to their spectral opportunities. The "Score" vector contains the classification in descending order and the "Ranking" vector contains the information based on the real distribution of the channels.

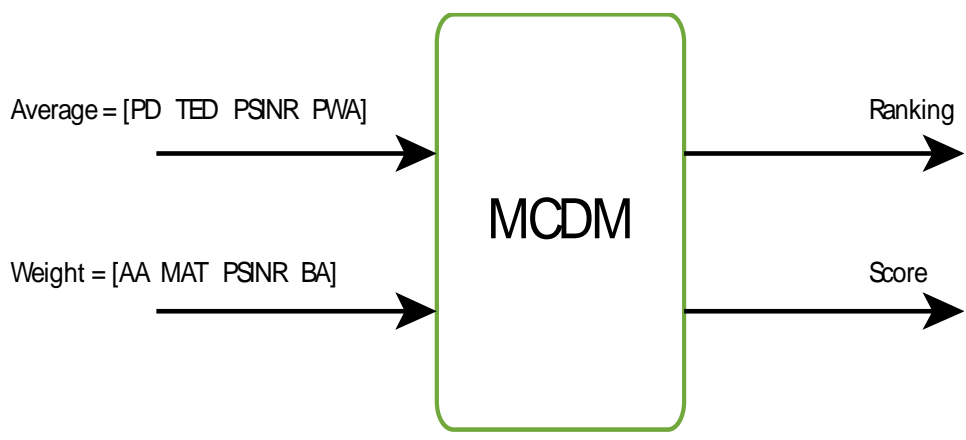

Figure 3. Inputs and outpus of the multi-criteria structure

For the implementation of the multi-criteria structures, two functions in Matlab are used. One for the SAW strategy and one for the FFAHP strategy. In the following pseudo-codes, the summary of the implemented algorithm is presented. The general structure consists on implementing the previously described mathematical models. 


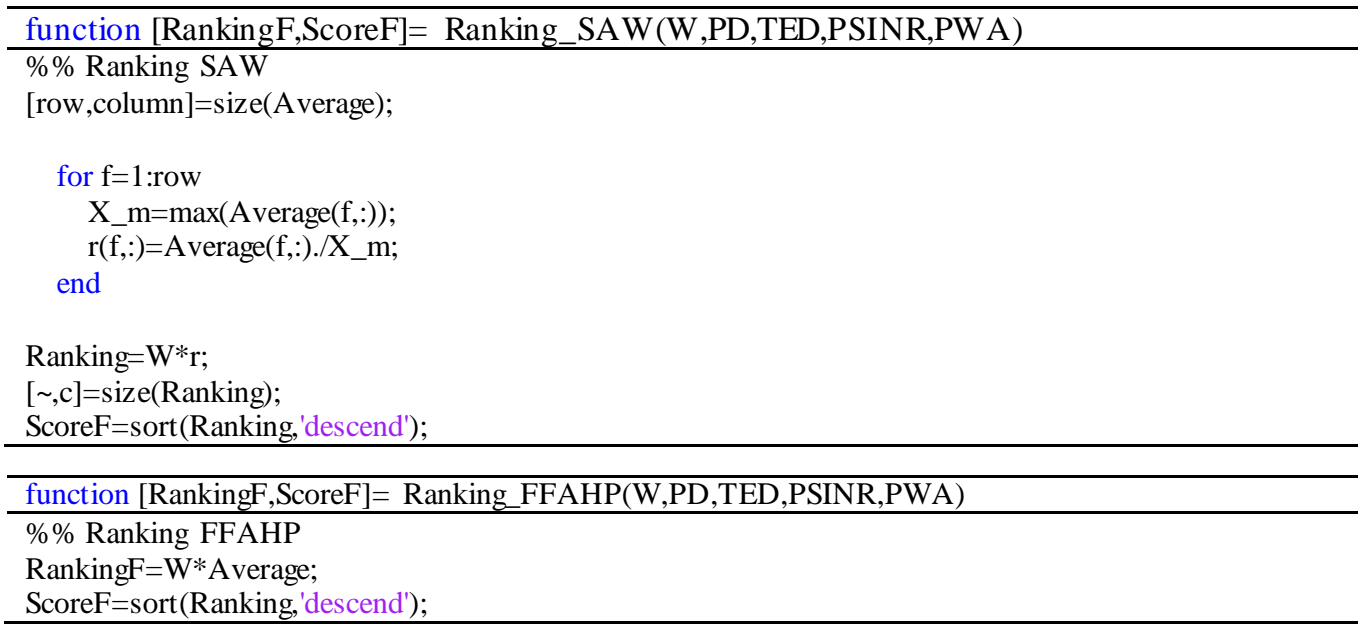

\subsubsection{Search algorithm}

In order to analyze throughput, a fourth algorithm is implemented to perform channel (frequency) hopping within the availability matrix according to the scores assigned by the multi-criteria module. If a channel is unavailable, it will then automatically search for the next channel and carry out the respective hopping operation. Each hop is quantified in a results matrix that finally leads to the behavior of throughput.

In Figure 1, the input variable "Time" represents the time instant and stop condition of the search algorithm, meaning that the algorithm hops from one row to another until the established time runs out. Furthermore, the model has an input variable called "Criteria Time" which establishes the time taken by the search algorithm to hop based on the previously computed position of the row-vector. If the stopping "Time" is higher than the "Criteria Time" when the search algorithm reaches time $t=$ criteria time, then the postion vector will be updated by recalculating the average values. The assessment is carried out through a training and validation matrix. The results correspond to the throughput figures for high and low traffic, with service classes RT and BE.

\section{RESULTS AND DISCUSSION}

To analyze the multi-criteria decision-making process (MCDM) with FFAHP and SAW, throughput is defined as an assessment metric in four different scenarios: GSM RT HT, GSM RT LT, GSM BE HT and GSM BE LT. The results obtained for FFAHP are presented in Figure 4 and the results for SAW in Figure 5. Ten simulations were performed for each experiment and then the average of each experiment was plotted.

In FFAHP scenarios, Figure 4 shows that the behavior throughout the 9 minutes of transmission is similar in all four types of cooperation, both for RT and BE. This hinders the importance of this variable within a spectral allocation model. The most significant variations are identified for models that use a $10 \%$ cooperation level. For SAW, Figure 5 shows that the evidenced similarity is maintained for RT and BE. In general, variations take place during the initial transmission times and the lowest throughput levels are obtained for the lowest cooperation levels.

Table 2 shows the relative values of the comparative performance evaluation in percentage form in the scenarios with different levels of cooperation. Although there is an improvement in performance for both algorithms as the level of cooperation grows higher, it is concluded that said improvement does not exc eed $10 \%$ in most cases. Therefore, it could be interesting to assess each algorithm comparatively in all scenarios, taking into account the highest and lowest levels of cooperation which are $10 \%$ and $100 \%$ respectively, as shown in Table 3. FFAHP has a clear dominance in three out of the four GSM scenarios. In RT-HT, SAW manages to relegate FFAHP-100 to third place, with a difference of only $11 \%$. However, both strategies take first place in average.

Table 2. Benchmarking by level of cooperation for AAT

\begin{tabular}{|c|c|c|c|c|c|c|c|c|c|}
\hline AAT & $\begin{array}{c}\text { GSM } \\
\text { BE LT }\end{array}$ & $\begin{array}{c}\text { GSM RT } \\
\text { LT }\end{array}$ & $\begin{array}{c}\text { GSM BE } \\
\text { HT }\end{array}$ & $\begin{array}{c}\text { GSM RT } \\
\text { HT }\end{array}$ & AAT & $\begin{array}{c}\text { GSM BE } \\
\text { LT }\end{array}$ & $\begin{array}{c}\text { GSM RT } \\
\text { LT }\end{array}$ & $\begin{array}{c}\text { GSM BE } \\
\text { HT }\end{array}$ & $\begin{array}{c}\text { GSM RT } \\
\text { HT }\end{array}$ \\
\hline FFAHP SU10 & 99,19 & 97,21 & 99,73 & 88,76 & SAW SU50 & 96,71 & 97,88 & 93,99 & 95,29 \\
\hline SAW SU10 & 95,43 & 93,36 & 93,93 & 95,26 & FFAHP SU80 & 99,7 & 99,77 & 99,96 & 88,85 \\
\hline FFAHP SU20 & 99,65 & 99,55 & 99,85 & 88,81 & SAW SU80 & 97,13 & 97,88 & 94 & 95,3 \\
\hline SAW SU20 & 95,97 & 97,03 & 93,96 & 95,28 & FFAHP SU100 & 100 & 100 & 100 & 88,92 \\
\hline FFAHP SU50 & 99,68 & 99,77 & 99,95 & 88,82 & SAW SU100 & 97,13 & 98,15 & 94,41 & 100 \\
\hline
\end{tabular}




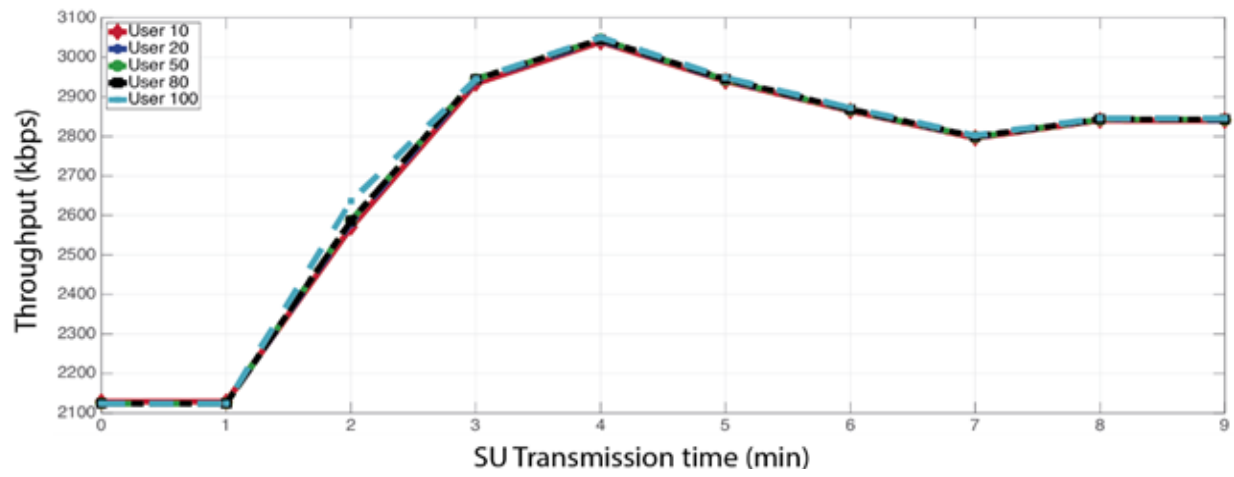

(a)

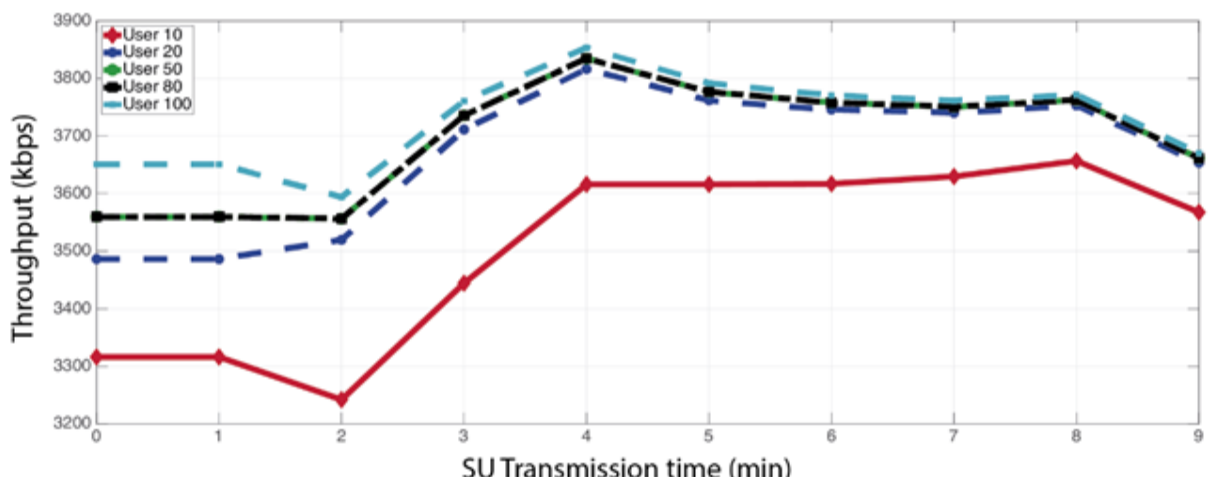

(b)

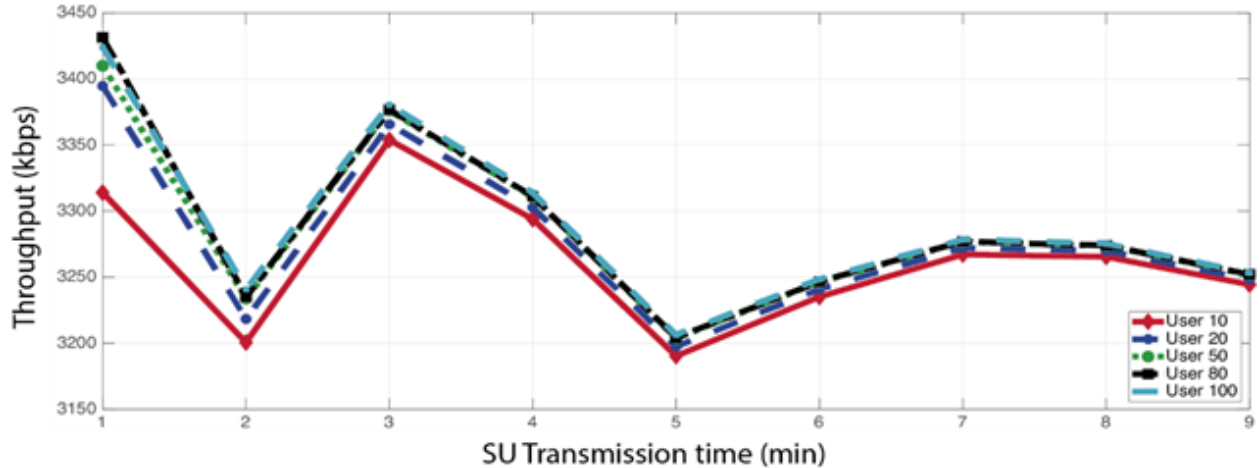

(c)

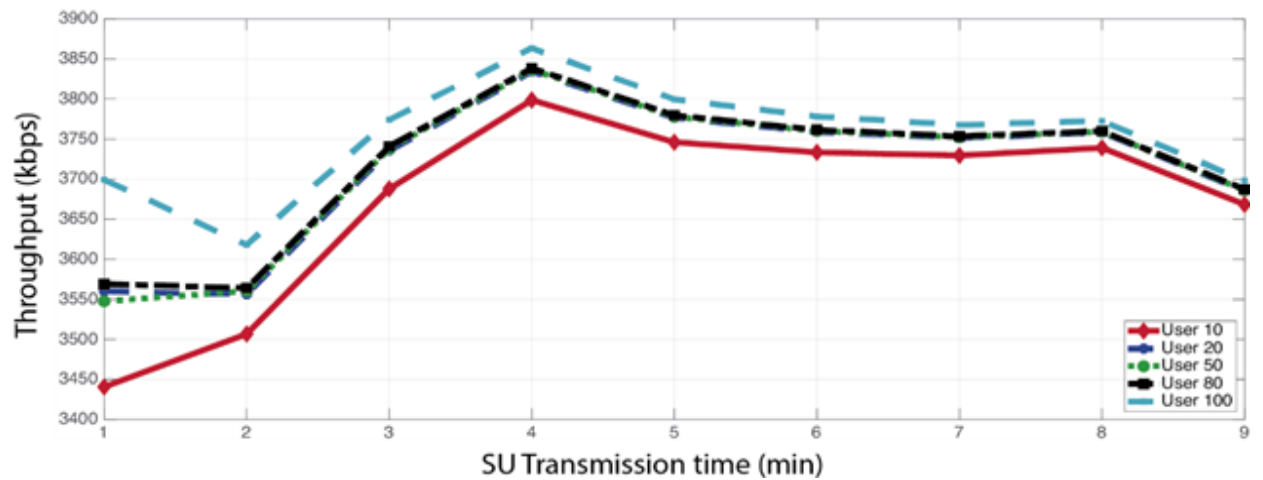

(d)

Figure 4. Throughput for FFAHP (a) GSM RT HT, (b) GSM RT LT, (c) GSM BE HT, (d) GSM BE LT 


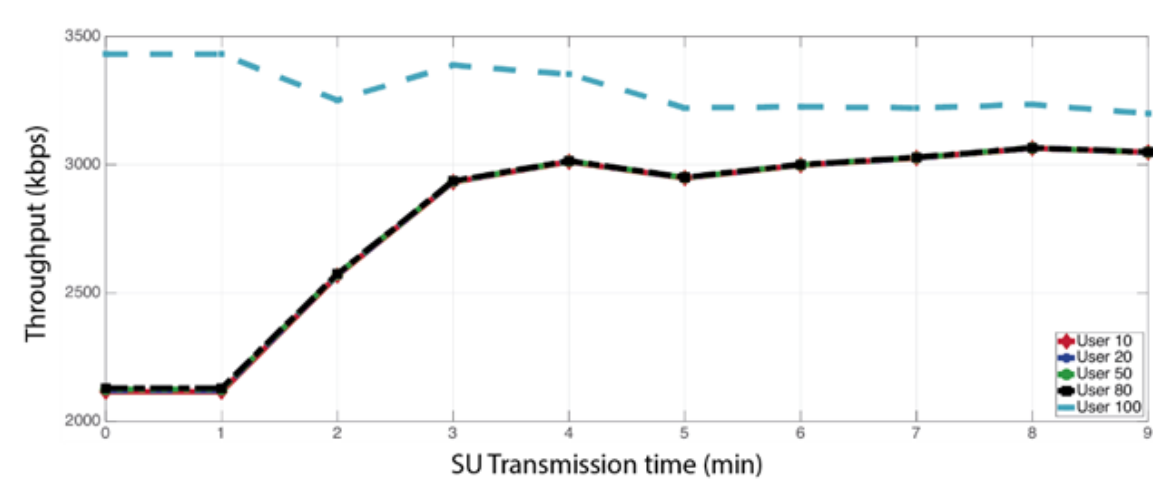

(a)

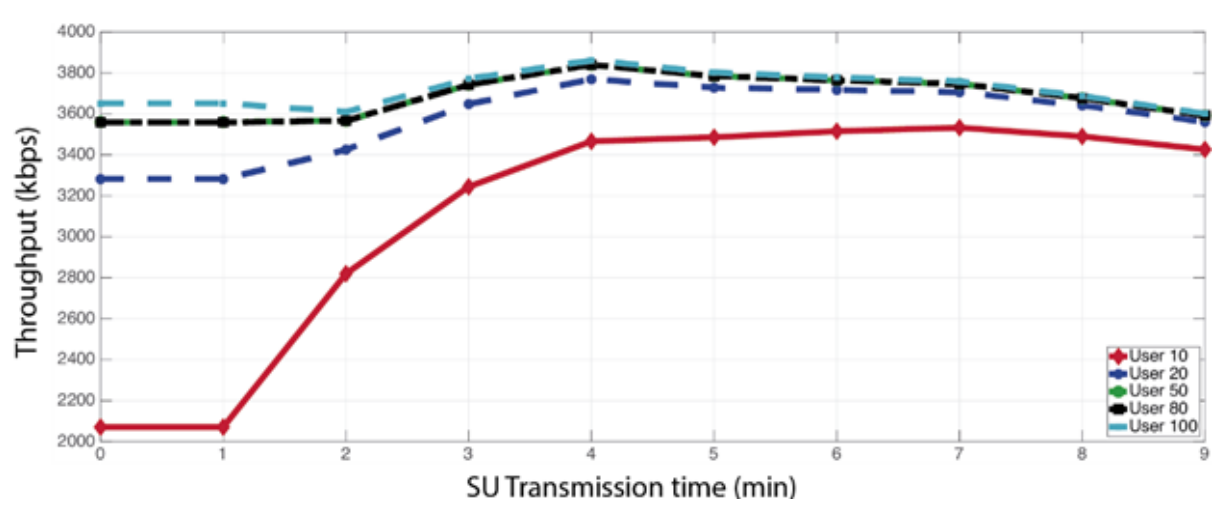

(b)

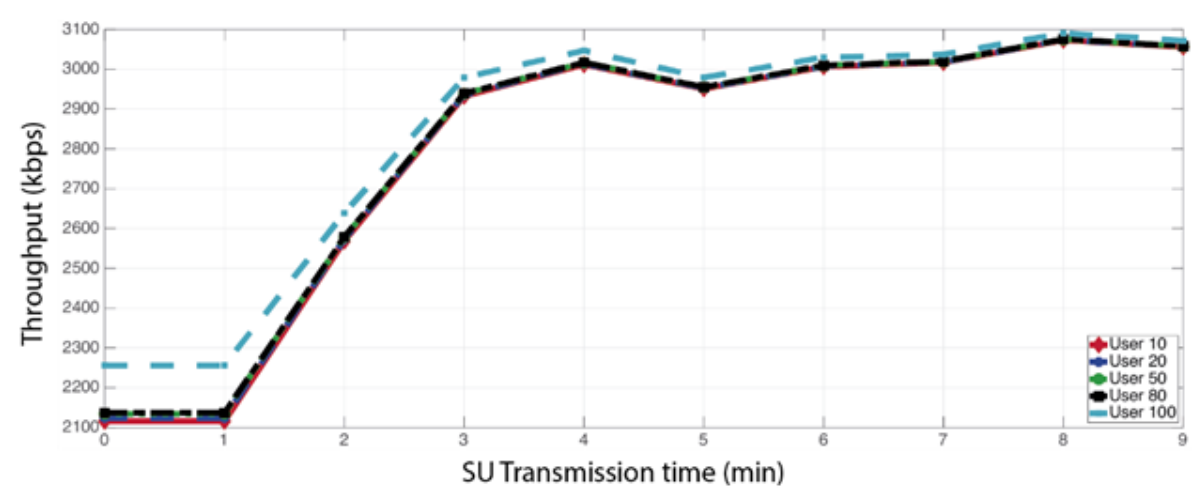

(c)

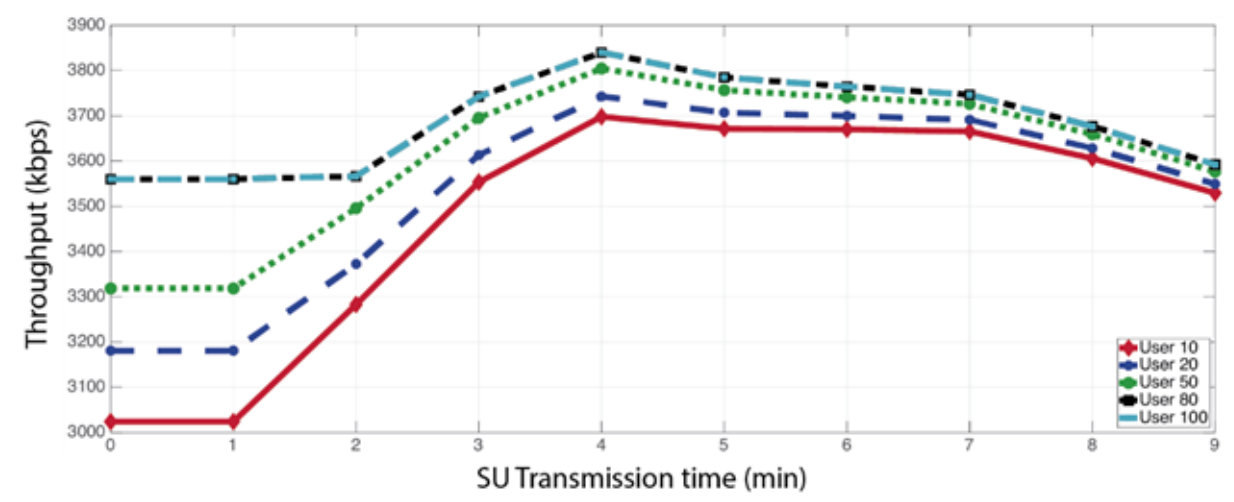

(d)

Figure 5. Throughput for SAW (a) GSM RT HT, (b) GSM RT LT, (c) GSM BE HT, (d) GSM BE LT 
At this point, the question is whether FFAHP-100 (FFAHP with a 100\% cooperation percentage) or FFAHP-10 (FFAHP with a 10\% cooperation percentage) is selected. By checking Table 3 once again, it is observed that FFAHP-100 has a performance of $98.23 \%$ and FFAHP-10 reaches 96.22\%, marking a slight difference of $2 \%$ in performance yet a significant difference of $90 \%$ in cooperation, which makes FFAHP-10 a better alternative.

Table 3. Benchmarking by scenario with $10 \%$ and $100 \%$ cooperation for AAT

\begin{tabular}{ccccc}
\hline AAT & FFAHP SU10 & SAW SU10 & FFAHP SU100 & SAW SU100 \\
\hline GSM BE LT & 99,19 & 95,43 & 100 & 97,13 \\
GSM RT LT & 97,21 & 93,36 & 100 & 98,15 \\
GSM BE HT & 99,73 & 93,93 & 100 & 94,41 \\
GSM RT HT & 88,76 & 95,26 & 88,92 & 100 \\
GSM LT & 98,2 & 94,395 & 100 & 97,64 \\
GSM HT & 94,245 & 94,595 & 94,46 & 97,205 \\
GSM BE & 99,46 & 94,68 & 100 & 95,77 \\
GSM RT & 92,985 & 94,31 & 94,46 & 99,075 \\
Score GSM & 96,222 & 94,495 & 98,23 & 97,422 \\
\hline
\end{tabular}

\section{CONCLUSION}

In cognitive radio networks, the decisions made by users are fairly limited which leads to an inability to properly use available spectrum resources even with dynamic access. The decision-making process in cognitive radio networks among multiple users is a challenge for next generation systems. When making the decision to access a channel, each secondary user should not only consider the quality of the channel but also the decisions regarding channel access is sued by other users. When more SU have access to the same channel, their performance is reduced due to mutual interference. In multi-user systems, cooperative strategies have delivered excellent results in decision-making, which allows an improvement of variables such as quality of service, latency, throughput, reliability, signalization, PU interference, energetic efficiency, bandwidth, SINR and error rate.

\section{ACKNOWLEDGEMENTS} this research.

The authors wish to thank Universidad Distrital Francisco José de Caldas for funding and supporting

\section{REFERENCES}

[1] C. Hernández, L. F. Pedraza, and E. Rodriguez-Colina, "Fuzzy Feedback Algorithm for the Spectral Handoff in Cognitive Radio Networks," Revista Facultad de Ingenieria, vol. 81, pp. 47-62, December 2016.

[2] B. Wang and K. J. R. Liu, "Advances in cognitive radio networks: A survey," in IEEE Journal of Selected Topics in Signal Processing, vol. 5, no. 1, pp. 5-23, Feb. 2011.

[3] C. Hernández, L. F. Pedraza, I. Páez, and E. Rodriguez-Colina, "Análisis de la Movilidad Espectral en Redes de Radio Cognitiva," Inf. tecnológica, vol. 26, no. 6, pp. 169-186, 2015.

[4] M. R. Ramzan, N. Nawaz, A. Ahmed, M. Naeem, M. Iqbal, and A. Anpalagan, "Multi-objective optimization for spectrum sharing in cognitive radio networks: A review," Pervasive Mob. Comput., vol. 41, pp. 106-131, 2017.

[5] M. A. Hazar, N. Odabasioglu, T. Ensari, Y. Kavurucu, and O. F. Say an, "Performance analy sis and improvement of machine learning algorithms for automatic modulation recognition over Rayleigh fading channels," Neural Computing and Applications, vol. 29, no. 9, pp.351-360, 2018.

[6] F. C. Committee, "In the matter of facilitating opportunities for flexible, efficient, and reliable spectrum use employ ing cognitive radio technologies, authorization and use of software defined radios," nprm 03-322, 2003.

[7] C. Hernández, I. Páez, and D. Giral, "Modelo AHP-VIKOR para handoff espectral en redes de radio cognitiva," Tecnura, vol. 19, no. 45, pp. 29-39, 2015.

[8] I. F. Akyildiz, W. Lee, M. C. Vuran and S. Mohanty, "A survey on spectrum management in cognitive radio networks," in IEEE Communications Magazine, vol. 46, no. 4, pp. 40-48, April 2008.

[9] I. F. Akyildiz, L. Won-Yeol, M. C. Vuran, and S. Mohanty, "NeXt generation/dynamic spectrum access/cognitive radio wireless networks: A survey," Comput. Networks, vol. 50, no. 13, pp. 2127-2159, 2006.

[10] Y. Cheng, E. H. Wu and G. Chen, "A Decentralized MAC Protocol for Unfairness Problems in Coexistent Heterogeneous Cognitive Radio Networks Scenarios With Collision-Based Primary Users," in IEEE Systems Journal, vol. 10, no. 1, pp. 346-357, March 2016.

[11] C. Salgado, S. Mora, and D. Giral, "Collaborative algorithm for the spectrum allocation in distributed cognitive networks," International Journal Engineering Technology, vol. 8, no. 5, pp. 2288-2299, 2016. 
[12] P. Thakur, A. Kumar, S. Pandit, G. Singh, and S. N. Satashia, "Spectrum mobility in cognitive radio network using spectrum prediction and monitoring techniques," Phys. Commun., vol. 24, pp. 1-8, 2017.

[13] G. I. Tsiropoulos, O. A. Dobre, M. H. Ahmed and K. E. Baddour, "Radio Resource Allocation Techniques for Efficient Spectrum Access in Cognitive Radio Networks," in IEEE Communications Surveys \& Tutorials, vol. 18, no. 1, pp. 824-847, Firstquarter 2016.

[14] D. A. López, E. R. Trujillo, and O. E. Gualdron, "Elementos Fundamentales que Componen la Radio Cognitiva y Asignación de Bandas Espectrales," Inf. tecnológica, vol. 26, no. 1, pp. 23-40, 2015.

[15] S. S. Oyewobi and G. P. Hancke, "A Survey of Cognitive Radio Handoff Schemes, Challenges and Issues for Industrial Wireless Sensor Networks (CR-IWSN)," Journal of Network Computer Applications, vol. 97, pp. 140-157, 2017.

[16] E. Ahmed, A. Gani, S. Abolfazli, L. J. Yao and S. U. Khan, "Channel Assignment Algorithms in Cognitive Radio Networks: Taxonomy, Open Issues, and Challenges," in IEEE Communications Surveys \& Tutorials, vol. 18, no. 1, pp. 795-823, Firstquarter 2016.

[17] A. Lertsinsrubtavee and N. Malouch, "Hybrid Spectrum Sharing through Adaptive Spectrum Handoff and Selection," in IEEE Transactions on Mobile Computing, vol. 15, no. 11, pp. 2781-2793, 1 Nov. 2016.

[18] K. Kumar, A. Prakash, and R. Tripathi, "Spectrum handoff in cognitive radio networks: A classification and comprehensive survey," Journal of Network Computer Applications, vol. 61, pp. 161-188, 2016.

[19] IEEE COMSOC, "IEEE Standard Definitions and Concepts for Dynamic Spectrum Access: Terminology Relating to Emerging Wireless Networks, Sy stem Functionality, and Spectrum Management," 2008.

[20] T. Kaya and C. Kahraman, "Multicriteria renewable energy planning using an integrated fuzzy VIKOR \& AHP methodology: The case of Istanbul," Energy, vol. 35, no. 6, pp. 2517-2527, 2010.

[21] C. Ramírez Pérez and V. M. Ramos Ramos, "Handover vertical: un problema de toma de decisión múltiple," in Congreso Internacional sobre Innovación y Desarrollo Tecnológico, 2010.

[22] C. Ramirez-Perez and V. Ramos-R, "On the Effectiveness of Multi-criteria Decision Mechanisms for Vertical Handoff," 2013 IEEE 27th International Conference on Advanced Information Networking and Applications (AINA), pp. 1157-1164, Barcelona, 2013.

[23] S. Liu, S. Pan, Z. Mi, Q. Meng and M. Xu, "A Simple Additive Weighting Vertical Handoff Algorithm Based on SINR and AHP for Heterogeneous Wireless Networks," 2010 International Conference on Intelligent Computation Technology and Automation, pp. 347-350, Changsha, 2010.

[24] E. Stevens-Navarro, R. Gallardo-Medina, U. Pineda-Rico, and J. Acosta-Elias, "Application of M ADM method VIKOR for vertical handoff in heterogeneous wireless networks," in IEICE Transactions on Communications, vol. 95, no. 2, pp. 599-602, 2012.

[25] C. Bernal and C. Hernández, "Modelo de decisión espectral para redes de radio cognitiva," Primera Ed. Bogotá, 2019. 